\title{
Review of: "Adapting the coordination of eyes and head to differences in task and environment during fully-mobile visual exploration"
}

\author{
Cédrick Bonnet
}

Potential competing interests: The author(s) declared that no potential competing interests exist.

Review of "Adapting the coordination of eyes and head to differences in task and environment during fullymobile visual exploration", Plos ONE, September $13^{\text {th }} 2021$.

Abstract: dear investigators. Thank you very much for such a remarkable publication. The last years, I searched such a paper discussing the coordination of free eye and head movements to perform freeviewing and search task that could fit with my results and this is exactly what I was looking for. So, thank you so much to provide a synthesis of the literature, two complementary studies and a clear and wellwritten message.

My main expertise is in postural control when participants perform precise task (search task) vs. unprecise tasks (free-viewing tasks) in the standing position. My other interest is in the interaction (synergy and also coordination) between eye movement, head-body movements when performing these visual tasks $\left(\sim 20^{\circ}\right)$ on small vs. large visual display $\left(100-120^{\circ}\right)$.

\section{Specific Comments}

With my expertise, I misunderstood some parts of your message and would like to suggest some suggestions/complementary aspects. These comments are only specific and minor.

The studies are great to show the way eye and head contribute to visual exploration of the environment both in walking alone and searching (walking and searching). A limitation in the study is that you did not explain reasons why people would turn their head more - in proportion - than they eyes. I noticed that you discussed "costs" but as far as I understand, this argument may not explain the results. In fact, the head is heavy and the fact of moving the head is costly. As everybody knows, the eyes are weightless and they can scan the environment extremely quickly. With this view, this is costlier to move more the head than the eyes to look at the same part of the environment. In your results, you clearly explained that the participants moved more their head than their eyes at each amplitude of gaze shifts in searching than in walking. Then, why the participants used more costly body (eye and head) movements in searching than walking is not explained. Consistently, I do not understand all references and explanation in the manuscript with such idea of cost:

- p. $22^{\text {nd }}$ half of the page: “When instructed to make two sequential gaze shifts, the head contributes 
more to the initial gaze shift if the second gaze shift will be in the same direction [22]. In other words, observers are more willing to rotate the head when the head will stay rotated for a while. This speaks to the different costs of eye versus head movements. The eyes can move quickly with little effort, whereas the head moves more slowly and requires more energy [12, 17].". If the head moves, then why discussing a reduced cost?

- p. 2 last paragraph: "Pelz and colleagues [16] instructed participants to copy a model, placed to the side of the participant, by arranging blocks on a workspace in front of the body. Participants turned their eyes and head to shift gaze between the model and workspace while completing the task. Unlike more controlled studies, the head contributed between 1ㅇ-10ㅇ for smaller gaze shifts (less than 15응 amplitude). Most likely, participants adapted eye and head rotations from moment to moment depending on the demands of looking to the model versus workspace (and scanning back and forth between the two locations). Participants' willingness to visually explore with eyes versus head may reflect the motor costs of each movement." I do not understand: do the participants move more or less in this study? Do they use more or less motor costs of each movement?

- p. $32^{\text {nd }}$ half of the page: "Even though the 140 horizontal spread is well below the oculomotor range of 50-55o, the head contributed to the horizontal spread of gaze: The horizontal spread of eye position was only 4-5, thus, the head accounted for the remaining portion". I do not understand why the head moved more than the eyes (about $9-10^{\circ}$ vs. $4-5^{\circ}$ ) if head movements are costlier than eye movement. "Similarly, Tomasi and colleagues [9] measured horizontal eye and head movements in walking participants using wearable inertial sensors, and found the head's rotation was responsible for between $37-46 \%$ of the total gaze shift amplitude across participants." Similarly, with your explanation of cost only, I do not understand why people move their head $37-46 \%$ of the total gaze shift amplitude. This result would only be consistent with the cost hypothesis if gaze shifts were large, is it the case in Tomasi et al ?

- p. 5 first paragraph: I like your hypothesis "we predicted that the head would contribute more to gaze shifts in the searching task to facilitate a wider spread of gaze in the environment?" However, we do not understand it on the basis of the cost hypothesis. You did not explain it, there is no reference coming to support it. So why did you have such a hypothesis?

- This conclusion is not helpful or useful for the moment (p. $142^{\text {nd }}$ half of the page): "Whether this is the most optimal or efficient strategy remains to be tested. Indeed, we cannot claim from the present work that energetic cost is the critical factor in shaping how eyes versus head contribute. Although head movements are more energetically costly, they also generate vestibular and proprioceptive information that eye movements do not." Indeed, what is your explanation in saying that "they also generate vestibular and proprioceptive information that eye movements do not"? Please develop here.

Overall, the hypothesis of cost should be better explained as I may misunderstand the message here.

In your manuscript, you only discussed the cost hypothesis to explain your results and I misunderstood this 
explanation (for me this is a counter-intuitive explanation). This is true that you proposed another explanation (the fact that head rotation "allows the eyes to stay within a more comfortable range of $\pm 25^{\circ}$. [21]" (p. $22^{\text {nd }}$ half of the page) but this is not a sufficient explanation. Indeed, what about studies in which the head turned while gaze only turned $<20^{\circ}$ ? For example, p. $32^{\text {nd }}$ half of the page (already suggested earlier): "Even though the 14은 horizontal spread is well below the oculomotor range of 50-55, the head contributed to the horizontal spread of gaze: The horizontal spread of eye position was only 40-50, thus, the head accounted for the remaining portion". In reading your manuscript, I strongly expected to find another explanation, i.e. an explanation related to a strategy to better perceive the environment. Unfortunately, I did not read this explanation. This explanation was only partially suggested as a question for future studies (p. $142^{\text {nd }}$ half of the page): "Future work could experimentally restrict head movement or increase the energetic cost of head movements to determine: 1) whether the eyes compensate by increasing their spread when head movement is reduced, and 2) whether a diminished contribution of the head to visual exploration degrades search performance." I would suggest another explanation for your results (instead of the cost hypothesis). Why not discussing an "eye centered strategy"?

In the literature on visual processes, many investigators already suggested that perception is best when the pupil is centered in the orbit (Proudlock and Gottlob 2007; Cui et al. 2010). As a larger number of cones are around the fovea, this position of the eyes is optimal to well perceive details around this position (Jonas et al. 1992). Furthermore, when the pupil is centered, the acquisition of future visual targets with gaze shifts is facilitated (Proudlock and Gottlob 2007). In the searching condition, your participants may have used an eye-centered strategy (i.e. they may have turned more their head to keep their pupil centered int he orbit) to optimize their performance in searching and retrieving. Your participants indeed spent more energy to turn their head proportionally more than in the walking condition, but they needed to better perceive their environment to succeed in the search task requested. In the searching condition, they needed to increase their chance to optimally perceive their environment and they did so. Of course, this explanation should be developed more extensively. Clearly I prefer this explanation than your counterintuitive explanation of cost.

\section{Minor Comments}

I also have some minor comments, coming from my single reading.

For me, study 1 is complementary. After reading the introduction, I absolutely did not understand why you performed a study only recording eye movements? Indeed, your goal and hypothesis related to eye and head movements and not only in eye movements. I was confused in reading Study 1 . After reading your manuscript, I understood that you could not put in $2^{\text {nd }}$ because it made no sense to do in $2^{\text {nd }}$. So, of course Study 1 should come first. But for the reader, it would be appreciated to have an introduction to explain the goal of this study and suggesting that there is no head movement. For the moment, this indication is only present in the title of Study 1, which is not sufficient for best understanding.

At the beginning of the introduction, I could read "Visual exploration refers to the active process of looking 
around in the environment. Observers survey the environment by shifting their gaze from one location to another ("scanning") to gather visual information that supports ongoing activities [1-3]." I agree that this is true but I would make the distinction between "visual exploration with no goal (for me this is not an active exploration)" and "visual exploration with a goal (e.g. searching). (for me this is an active exploration). I have no legitimacy to say so as many investigators already suggested that Visual exploration refers to the active process of looking around in the environment" but I really believe that freeviewing the environment with no goal is not an active exploration. At least my results in my synergic studies (Bonnet et al., 2017, 2019, 2019, 2021) all support this idea. This was just for noticing.

p. 5/17 first line. "the" is repeated twice. The same problem is visible p. 14/17 6 lines before the end of the page.

In the transition (first paragraph Study 2 p. 10). I suggest to invert the two coming sentences: "It is possible that the more extreme rotations of the eyes during the search task were oppositional movements to compensate for head rotation. If so, the observer would not truly be spreading gaze more in the searching task compared with the walking task. Alternatively, if participants in the search task rotated their eyes and heads more in the same direction at the same time, then the spread of gaze when searching would truly be greater." Indeed, as a reader, I expected the second aforementioned idea (head supporting the eyes movements) and therefore waited to read this sentence first. The other possibility (head movements opposed to eye movements) is certainly possible but unexpected for me (why would people do so?).

The full paragraph "However, given the flexibility of coordinating eyes, head, and body, an increase in spread of gaze while searching could be accomplished in different ways: a larger spread of eye position without a change in head position, a larger spread of head position without a change in eye position, or increasing spread of both eyes and head. One possibility is that observers rotate the head more broadly to search in areas to the left and right of the current walking direction beyond the range of the eyes. Another possibility to rule out, however, is whether observers avoid extreme head rotations while searching if it disrupts their ability to guide locomotion. If so, we would observe an increase in the spread of eye movements but not head movements. It is important to note that we make no specific claim about the extent to which changes in the spread of eye or head movements might reflect conscious decision making. Although it is true that observers can consciously choose to employ greater head versus eye movements while exploring, it seems more likely-especially while engaged in a task like searching - that participants are not consciously deciding moment-to-moment how much to move the eyes versus head. Regardless, the current studies were not designed to distinguish between these possibilities." Is extremely long and useless as next you make the relevant hypothesis: "we predicted that the head would contribute more to gaze shifts in the searching task to facilitate a wider spread of gaze in the environment." Instead, I would have preferred to read this hypothesis with arguments and citations going in this direction.

p. 3 second half of the page: "Other studies of eye movements while walking over flat ground consistently find a larger horizontal than vertical spread of eye position: 14.2 versus 9.70 [30], 70 versus 50 [7], and 
11.8 versus $7.2^{\circ}$ [5]." This sentence is decontextualized. It should have come earlier in the introduction when you discussed amplitude of eye movements in horizontal and vertical directions.

For the presentation of the results in the two studies, I do not understand why you presented the results in the walking condition, then in the searching condition before writing the statistical analyses? Why not writing the statistical analysis first before only writing in which of the two conditions the dependent variable was higher or lower? In this way you would not repeat the same result higher in one condition and (therefore) lower in the other condition. 